\title{
The Natural History of Egg Allergy in an Observational Cohort
}

\author{
Scott H. Sicherer, MD ${ }^{1,{ }^{*}}$, Robert A. Wood, MD2, ${ }^{*}$, Brian P. Vickery, MD $^{3}$, Stacie M. Jones, \\ $\mathrm{MD}^{4}$, Andrew H. Liu, MD ${ }^{5}$, David M. Fleischer, MD $^{5}$, Peter Dawson, PhD $^{6}$, Lloyd Mayer, \\ $\mathrm{MD}^{1,{ }^{1 *}}, \mathrm{~A}$. Wesley Burks, $\mathrm{MD}^{3}$, Alexander Grishin, $\mathrm{PhD}^{1}$, Donald Stablein, $\mathrm{PhD}^{6}$, and Hugh \\ A. Sampson, MD ${ }^{1}$ \\ ${ }^{1}$ Department of Pediatrics, Icahn School of Medicine at Mount Sinai, New York, NY \\ ${ }^{2}$ Department of Pediatrics, Johns Hopkins University School of Medicine, Baltimore, MD \\ ${ }^{3}$ Department of Pediatrics, University of North Carolina, Chapel Hill, NC \\ ${ }^{4}$ Department of Pediatrics, University of Arkansas for Medical Sciences and Arkansas Children's \\ Hospital, Little Rock, AR \\ ${ }^{5}$ Department of Pediatrics, National Jewish Health, Denver, CO \\ ${ }^{6}$ The EMMES Corporation, Rockville, MD
}

\section{Abstract}

Background-There are few studies on the natural history of egg allergy and most are single site, not longitudinal, and have not identified early predictors of outcomes.

Objective-To describe the natural course of egg allergy and to identify early prognostic markers.

\begin{abstract}
Methods-Children aged 3-15 months were enrolled in a multicenter observational study with either a convincing history of an immediate allergic reaction to egg and/or milk with a positive prick skin test (SPT) to the trigger food; and/or moderate-severe atopic dermatitis and a positive SPT to egg or milk. Children enrolled with a clinical history of egg allergy were followed longitudinally and resolution was established by successful ingestion.
\end{abstract}

\begin{abstract}
Results-The egg-allergic cohort consists of 213 children followed to a median age of 74 months. Egg allergy resolved in 105 (49.3\%), at a median age of 72 months. Factors that were most predictive of resolution included the following: initial reaction characteristics (isolated urticaria/angioedema vs other presentations), baseline egg-specific IgE level, egg SPT wheal size, atopic dermatitis severity, IgG4 and IL-4 response (all $\mathrm{P}<0.05$ ). Numerous additional baseline clinical and demographic factors and laboratory assessments were not associated with resolution. Multivariate analysis identified baseline egg-specific $\mathrm{IgE}$ and initial reaction characteristics as strongly associated with resolution; a calculator to estimate resolution probabilities using these variables was established.
\end{abstract}

\footnotetext{
C 2013 American Academy of Allergy, Asthma and Immunology. Published by Mosby, Inc. All rights reserved.

Corresponding Author (and reprint requests): Scott H. Sicherer, MD, Division of Allergy/Immunology, Mount Sinai Hospital, Box 1198, One Gustave L. Levy Place, New York, New York 10029-6574. Voice: (212) 241-5548; FAX: (212) 426-1902; scott.sicherer@mssm.edu.

**is. Sicherer and Wood contributed equally to this manuscript

Deceased.
}

Publisher's Disclaimer: This is a PDF file of an unedited manuscript that has been accepted for publication. As a service to our customers we are providing this early version of the manuscript. The manuscript will undergo copyediting, typesetting, and review of the resulting proof before it is published in its final citable form. Please note that during the production process errors may be discovered which could affect the content, and all legal disclaimers that apply to the journal pertain. 
Conclusions-In this cohort of infants with egg allergy, about one half had resolved over 74 months of follow-up. Baseline egg-specific IgE and initial reaction characteristics were important predictors of the likelihood of resolution.

\section{Keywords}

egg allergy; natural history; food allergy; IgE

\section{INTRODUCTION}

Allergy to egg is estimated to affect $0.5-2.5 \%$ of young children, ${ }^{(1-4)}$ with a recent estimate of up to $8.9 \%$ of infants reacting to raw egg in one study from Australia. ${ }^{(5)}$ Having an egg allergy, or being sensitized to egg, is associated with increased risks of peanut and other food allergies, atopic dermatitis (AD) and development of respiratory allergies and asthma. ${ }^{(6-9)}$ For those with egg allergy, avoidance is difficult and allergic reactions from accidental ingestion are common. ${ }^{(10)}$ Fortunately, egg allergy typically resolves during childhood. ${ }^{(11-14)}$ However, the rate of resolution may be slowing, with a past study suggesting the majority are egg tolerant by age $3 \mathrm{yrs}^{(13)}$ and a recent study suggesting about half of children reach tolerance by age $12 \mathrm{yrs} .{ }^{(11)}$ The recent study from a referral population showed persistent egg allergy for $42 \%$ of children in late adolescence, ${ }^{(11)}$ suggesting the number of egg allergic adults may increase with time, although the current estimate of egg allergy among adults is $0.2 \%$. $^{(9)}$ The ability to determine prognosis of egg allergy is critical because potential interventions under study carry risks ${ }^{(15)}$ and ideally would be applied in those unlikely to achieve resolution naturally.

The Consortium of Food Allergy Research (CoFAR) enrolled 512 infants with likely egg or milk allergy, but without previously known peanut allergy, in a multicenter observational study to address the immunologic, genetic, and environmental factors that affect the natural course of food allergy. ${ }^{(16 ; 17)}$ Evaluations were offered every 6 months and oral food challenges (OFC) were offered as clinically indicated, similar to the studies described above..$^{(1-14)}$ We previously reported the natural course of milk allergy in this cohort and identified a number of prognostic markers that could be used to estimate resolution rates utilizing baseline characteristics. ${ }^{(18)}$ The primary aim of the current analysis is to assess the natural history of egg allergy in the infants enrolled in this cohort, with a focus on the clinical factors predicting the resolution of egg allergy over the first six years of life.

\section{METHODS}

\section{Subjects, study definitions and procedures}

The subjects of this study are an egg-allergic subset of a larger cohort of 512 infants originally enrolled at 3-15 months of age at 5 sites (egg allergic children/total enrolled per site): The Icahn School of Medicine at Mount Sinai, New York (47/106); Duke University Medical Center, Durham, NC (now followed at the University of North Carolina, 20/103); Johns Hopkins University School of Medicine, Baltimore, MD (37/109); National Jewish Health, Denver, CO (42/99), and Arkansas Children's Hospital, Little Rock, AR (67/95) as described previously. ${ }^{(16 ; 18)}$ Enrollment criteria for the whole cohort were designed to include atopic children with likely egg or milk allergy at risk to develop peanut allergy, but without current peanut allergy. Briefly, enrollment required either (1) a history of a convincing immediate allergic reaction to egg and/or cow's milk and a positive skin prick test (SPT) to the trigger food, and/or (2) moderate to severe AD, and a positive SPT to egg and/or milk. Exclusion criteria included clinical evidence of peanut allergy or peanutspecific $\operatorname{IgE}>5 \mathrm{kU}_{\mathrm{A}} / \mathrm{L}$ identified prior to enrollment. ${ }^{(16 ; 18)}$ 
The subgroup of children in the current study had a diagnosis of egg allergy at the time of enrollment, or acquired this diagnosis after enrollment with no prior evidence of egg tolerance. Study procedures were reviewed and approved by the National Institutes of Allergy and Infectious Diseases Data Safety Monitoring Board and by local site Institutional Review Boards, and written signed consents were obtained.

Participants were considered egg-allergic if their initial reaction was either (1) a positive physician-supervised OFC; (2) a convincing reaction (defined by symptoms within an hour of isolated ingestion that included at least urticaria and/or angioedema, difficulty breathing, wheezing, throat tightness, and/or vomiting) AND sensitization to egg (egg-specific IgE $\searrow 0.35 \mathrm{kU}_{\mathrm{A}} / \mathrm{L}$ and/or SPT $\geq 3 \mathrm{~mm}$ ); or (3) a flare of AD associated with egg ingestion AND an egg-specific IgE level greater than $2 \mathrm{kU} / \mathrm{A}$, a level which is $>95 \%$ predictive of egg allergy in infants. ${ }^{(13)}$ Reaction details were recorded regarding skin, oral, respiratory, gastrointestinal and cardiovascular symptoms. The study analyzed 3 mutually exclusive initial clinical presentations of reaction to egg ingestion: $\mathrm{AD}$ diagnosis (flare of $\mathrm{AD}$ ), skin only (acute hives and/or angioedema) or systemic (e.g., more than isolated skin, including respiratory and gastrointestinal reactions). Subjects were considered egg tolerant if they ingested whole, concentrated egg products (scrambled egg, French toast) in serving size quantities without symptoms either during physician-supervised OFCs or after introduction at home. Dietary ingestion of products with extensively heated egg (baked egg, for example as an ingredient in a muffin or cookie) was queried but was not considered evidence of resolved egg allergy.

Dietary, medical and social histories were obtained using questionnaires completed during enrollment interviews. A diagnosis of asthma and allergic rhinitis was based on parental report or parental report of physician diagnosis. A diagnosis of other food allergies included per protocol definitions for egg and peanut, ${ }^{(16 ; 18)}$ while for other foods this was based on a clinical diagnosis by a study physician.

Diagnosis of baseline $\mathrm{AD}$, in distinction to $\mathrm{AD}$ flares due to egg ingestion described above, required pruritus and an eczematous rash (acute, subacute, chronic) with typical morphology and age-specific patterns, a chronic or relapsing history, atopy (personal and/or family history or IgE reactivity) and xerosis. AD severity was graded by criteria previously described and published by Rajka and Langeland. ${ }^{(19)}$ Briefly, the AD severity was graded as mild, moderate, or severe (Supplemental Table E1) as described previously. ${ }^{(18)}$. Atopic disease history in parents of the enrolled infants was based upon previously published definitions and was recorded by parental report. ${ }^{(20)}$

To maintain uniformity and an observational approach, the study design includes evaluations, care for food allergy, and instructions on dietary management that were uniform among the clinical centers, and reflect practice parameters in force at the time of enrollment for $\mathrm{AD},{ }^{(21)}$ food allergy, ${ }^{(22)}$ and allergy prevention. ${ }^{(23)}$ Participants were evaluated in person at enrollment, 6 months, 12 months and yearly thereafter, with additional telephone followup between each visit and instructions to contact the study site for any allergic reactions, at which time additional details were obtained. ${ }^{(10)}$ OFCs to egg were typically offered when egg-specific $\mathrm{IgE}$ serum concentrations were $\leq 2 \mathrm{kU} \mathrm{A}_{\mathrm{A}} / \mathrm{L}$ and skin test mean wheal diameter was $<10 \mathrm{~mm}$ if there was no reaction in the preceding 6 months. However, OFC was not withheld if additional clinical data warranted OFC outside of these parameters (e.g., tolerance of a small accidental exposure or parental preference). OFCs were considered positive for persistent subjective or for objective symptoms. ${ }^{(24)}$ OFCs were performed with cooked whole or pasteurized powdered egg, not raw egg white. 


\section{Skin Prick Tests (SPTs)}

SPTs were performed using the GreerPick ${ }^{\circledR}$ (Lenoir, NC) with participants avoiding antihistamines for at least 5 half-lives of the specific agent. Tests were performed on the infant's back, and at 15 minutes the wheal was outlined in pen and transferred by tape to paper. The size of the longest diameter and its longest perpendicular were averaged. A SPT score was computed by subtracting the saline control measure and a positive SPT is defined by a score of $3 \mathrm{~mm}$ or greater. Tests were considered reliable if the wheal of the negative control (50\% glycerin-saline) was $3 \mathrm{~mm}$ or smaller and wheal size of the histamine control was at least $3 \mathrm{~mm}$ larger than the wheal size of the negative control. All sites used the same lot of reagents, and training was performed to ensure consistency. The egg (chicken) white extract was obtained from Greer (catalog number F272).

\section{Serum egg-specific IgE and IgG4}

The concentration of specific IgE antibody to egg white was measured from plasma at a central laboratory (Mount Sinai) using the Thermo-Fisher ImmunoCAP® system (Uppsala, Sweden) reported in $\mathrm{kU}_{\mathrm{A}} / \mathrm{L}$. A level $>0.35 \mathrm{kU}_{\mathrm{A}} / \mathrm{L}$ was considered positive. The concentration of IgG4 antibodies to milk were also measured from plasma samples using the ImmunoCAP® system (detection limit $0.07 \mathrm{mg} / \mathrm{L}$ ).

\section{Mononuclear cell stimulation and PCR analysis}

Studies were performed to determine if egg-specific Th2 or T regulatory cell gene expression was predictive of egg allergy outcomes. Peripheral blood mononuclear cell isolation was performed by Ficoll-Paque density gradient centrifugation and cultures were performed at each clinical site on fresh venous blood samples as previously described. ${ }^{(16)}$ Briefly, 4 million cells per condition were cultured for 48 hours in AIM-V serum-free media (Invitrogen) with egg white protein $(50 \mathrm{mcg}$ each $/ \mathrm{ml})$, aqueous peanut extract $(50 \mu \mathrm{g} / \mathrm{ml})$, tetanus toxoid $(5 \mu \mathrm{g} / \mathrm{ml})$, purified $\alpha, \beta$, and $\kappa$-caseins $(50 \mu \mathrm{g}$ each $/ \mathrm{ml})$, and additional control stimulations were performed with medium alone (negative) and anti-CD3/-CD28 beads (positive). At the end of the culture period, cells expressing CD25 were enriched by selection with anti-CD25 coated para-magnetic beads according to the manufacturer's protocol (Miltenyi). Pilot experiments demonstrated $\sim 10$-fold enrichment of CD25+ cells with $70-80 \%$ of selected cells co-expressing CD3, CD4 and CD25 as measured by flow cytometry. The entire selected fraction of cells was immediately lysed in RLT buffer (Qiagen) and stored at $-80^{\circ} \mathrm{C}$ until RNA purification. The quantitative polymerase chain reaction (qPCR) was carried-out in the central laboratory according to the in-house established protocol utilizing SYBR Green I fluorescence detection in a 384 well plate on ABI 7900 (Applied Biosystems). Raw PCR analysis and annotation were performed on coded samples. Threshold cycle number $(\mathrm{Ct})$ was set by software with confirmation and adjustment as necessary to define the threshold of linear amplification. For the gene expression data, ddCt was calculated by subtracting the RPS9 reporter gene $\mathrm{Ct}$ and then normalizing by subtracting the standardized medium control response. Negative values indicate relatively higher activity with a unit score change corresponding to a doubling. Non-detected genes were arbitrarily assigned a Ct of 40 .

\section{Statistical analysis}

Time to resolution of egg allergy was measured with age as the time metric, whether enrolled with egg allergy or having the diagnosis following enrollment. While the time of allergy diagnosis varied depending on when food introduction and diagnostic testing were performed, each individual's first definitive diagnosis was positive for egg allergy. Proportional hazards regression models were fit to examine covariates for their impact on the hazard or risk function. ${ }^{(25)}$ The estimated survival distribution was calculated from the 
relative hazard, which is the exponentiated sum of the linear combination of the products of the parameter estimates with their respective clinical characteristics. The common underlying empirical cumulative hazard function Lambda(t) is estimated with a step function and the resolution curve is estimated as 1-exp(-RH*Lambda(t)). In this paper, hazard refers to the chance of a beneficial event, i.e., allergy resolution, and variables are structured so that large relative hazard values are associated with increased chance of allergy resolution. A multivariate proportional hazards model was fit using significant baseline factors from the univariate models to assess the probability of allergy resolution over time. The final model was selected based on factor significance and model fit. Time varying clinical covariate analyses used the most recent available assessment in the model and nonproportional hazards were examined by fitting linear and spline function interactions with time. Reported p-values are two-tailed when applicable and SAS 9.2 and R were used for the computations.

\section{RESULTS}

Of the 512 enrolled infants, the egg-allergic cohort consists of 213 children, of whom 140 were diagnosed with egg allergy at baseline. In the remaining 73 , the diagnosis was categorized as uncertain at their entry visit but egg allergy was subsequently confirmed at a median age of 23.2 months (interquartile range, $16.1-41.9$ months), 10 by OFC. Key baseline characteristics are summarized in Table 1 and Table E2. AD was present in 196, categorized as mild in 31, moderate in 104, and severe in 61. Twenty-seven infants (12.7\%) were diagnosed with egg allergy based on AD criteria while the remainder had a history of an acute reaction and positive tests. Ninety-three subjects were first diagnosed based on a reaction or clinical history that was limited to skin symptoms (hives, pruritus or swelling) after exposure. Another 93 subjects were diagnosed based on a reaction that involved more extensive symptoms (e.g., oral, upper/lower respiratory, GI or cardiovascular), in addition to or apart from urticaria/angioedema.

One hundred five of the 213 participants (49.3\%) have now resolved their egg allergy with a median age of resolution of 72 months and a median age at last follow-up of 74 months (Figure 1). Resolution was defined by OFC in $47(44.8 \%)$ and by successful home introduction of whole (not baked) egg products in the remainder, recorded by the time of their visit. Regarding exposure to egg in baked goods, at the 6 year time point, 43 of 113 $(38.1 \%)$ with unresolved allergy reported tolerating at least some baked egg products, while 4 reported reactions to ingestion of baked egg products.

Additional baseline characteristics of the cohort, comparing those with and without egg allergy resolution, are presented in Table 1 and Cox regression analyses are shown in Table 2. The baseline characteristics that were most predictive of egg allergy resolution included egg-specific IgE level and the characteristics of the presenting reaction. Specifically, highly significant differences $(\mathrm{P}<0.001)$ in the rate of resolution were noted when comparing those subjects with baseline egg-specific IgE levels $<2 \mathrm{kU}_{\mathrm{A}} / \mathrm{L}, 2-10 \mathrm{kU}_{\mathrm{A}} / \mathrm{L}$, and $\geq 10 \mathrm{kU}_{\mathrm{A}} / \mathrm{L}$ (Figure 2). Significant differences $(P=0.007)$ in resolution were also predicted by reaction classification, with those having acute reactions with only skin symptoms having a greater likelihood of resolution compared to those with acute reactions involving systems beyond the skin (Figure 3, distinguishing the 3 clinical categories described above). Those with an $\mathrm{AD}$ flare from egg and those with systemic reactions to egg had poorer prognosis than those with isolated urticaria/angioedema. The poor prognosis of the former reaction category may be partly influenced by the requirement for elevated egg specific IgE antibodies in the definition of an egg allergic reaction manifested by an AD flare. Resolution was also associated with baseline AD severity (Table 2 and supplemental Figure E1), egg-SPT (Table 2 and supplemental Figure E2) and egg-specific IgG4 (Table 2 and supplemental Figure E3) 
but only weakly. Only 18 (17.1\%) of the subjects with resolved egg allergy reported no AD at the time of resolution.

Additional factors that correlated with rapidity of resolution included gender, $\mathrm{T}$ cell responses (see below), and the egg-specific $\mathrm{IgE}$ to $\mathrm{IgG}_{4}$ ratio (hazard ratio $0.62(95 \% \mathrm{C}$, 0.47 to $0.82, p<0.001)$, although this latter effect appears to be a result of the strong correlation (Spearman $r=0.63$ ) with egg-specific IgE. Parameters not associated with resolution included race, breastfeeding, other food allergies, baseline milk allergy diagnosis, asthma or rhinitis, family income, parental education, presence of siblings, and parental atopy. Although baseline milk allergy was not related to egg allergy resolution, when resolution of milk allergy was examined as a time-varying covariate, it was associated with egg allergy resolution, and the effect persisted when adjusted for $\log$ egg IgE and skin reaction classification (data not shown).

Not surprisingly, baked egg (e.g., in muffins, cookies) consumption was related to resolution outcomes. ${ }^{(26)}$ Egg allergy resolution rates were 75/166 (45.2\%), 8/14 (57.1\%) and 17/24 (70.8\%) among the 204 cases reporting no baked egg, baked egg consumption with reaction and baked egg consumption without reaction at the 6 month follow-up visit. The instantaneous risk ratios for resolution are 1.8 and 3.4 for the latter classes versus the nonconsumption group, the difference is statistically significant $(\mathrm{p}<0.001)$ and is maintained after adjustment for $\log \operatorname{IgE}$ and skin reaction classification. At 6 years of age, baseline characteristics including reaction characteristics and egg-specific IgE levels did not predict those who would go on to ingest products with egg baked into them.

$\mathrm{T}$ cell studies were assessed at baseline for relationships of egg allergy resolution to antigen and control stimulated expression of mRNA for CISH, FOXP3, GATA3, TBET, IL-10, IL-4, and / or IFN- $\gamma .{ }^{(16)}$ Genes that were associated with egg allergy resolution (lower expression of these genes was associated with a greater chance of resolution) include IL4 stimulated by egg white (hazard ratio, HR=1.04, p-value $=0.047$, Supplemental Figure E4), peanut $(\mathrm{HR} 1.05$, $\mathrm{p}$-value $=0.037)$, tetanus $(\mathrm{HR}=1.06$, $\mathrm{p}$-value $=0.005)$, and casein $(\mathrm{HR}=1.06$, p-value $=0.005)$, as well as FOXP3 stimulated by casein $(\mathrm{HR}=1.05$, $\mathrm{p}-$ value $=0.046$ ). In adding egg-stimulated IL4 expression to the variables in the clinical resolution model (described below), the HR was 1.04, $\mathrm{p}=0.06$. Results for tetanus (HR $1.05, \mathrm{p}=0.024$ ) and casein (HR 1.05, $\mathrm{p}=0.01$ ) stimulated IL4 remained significant when added to the clinical model, but added little additional predictive information.

Finally, we used the 2 baseline factors most predictive of egg allergy resolution to develop a composite score that could be applied to individual patients (Supplemental Table E3). For example, as represented in Supplemental Figure E5, the likelihood of egg allergy resolution for 5 individual patients is predicted using a composite index incorporating their eggspecific IgE level and classification of reaction. An interaction between time and baseline egg IgE is observed, such that the predictive utility of baseline egg-specific IgE decreases at later time points. We have provided a web-based calculator based on our data, which is available for use in validation studies in other centers. (see www.cofargroup.org).

\section{DISCUSSION}

Here we described the natural history of egg allergy in a cohort of children enrolled in an observational study with a diagnosis of egg allergy. We found a resolution rate of almost $50 \%$ through age 6 years, which was similar to but slightly slower than the resolution rate of milk allergy in this cohort, which was approximately $50 \%$ by age 5 years. ${ }^{(18)}$ This result appears to be slightly less favorable than that reported in 58 children by Boyano-Martinez and colleagues, ${ }^{(13)}$ who found resolution rates of $50 \%$ by age $4-4.5$ years and $66 \%$ by age 7 
years in children referred with food allergy, $50 \%$ of whom had AD. Other studies report early childhood resolution rates from $31-51 \%$, but none are comparable due to different ages at presentation, referral bases and length of follow-up. ${ }^{(12 ; 14 ; 27 ; 28)}$ In another referral population to a tertiary care center reported by Savage and colleagues ${ }^{(11)}$ of 881 children, resolution by age 4 years was noted in only $4 \%$ and by age 8 years it was $26 \%$, worse than observed in the present cohort. Studies of egg (and milk) allergy resolution rates clearly vary by population, with referral populations showing slower resolution rates than less selected groups. ${ }^{(12 ; 29)}$ The specific predictors of egg allergy resolution were different from those we observed with milk in this same cohort (e.g., differences in influence of AD severity, gene expression profiles), ${ }^{(18)}$ which adds to additional previous observations regarding the uniqueness of egg allergy and sensitization compared to milk. For example, egg allergy is a stronger early indicator of future allergic reactivity, ${ }^{(30)}$ shows different gene expression profiles for allergic infants, ${ }^{(16)}$ and has a higher likelihood to induce anaphylaxis in baked foods ${ }^{(31)}$ compared to milk.

Few studies have attempted to identify early prognostic markers of egg allergy resolution, particularly prospectively. We identified a number of baseline factors that were associated with egg allergy resolution, but in the multivariate analysis, the characteristics of the presenting reaction and the egg-specific IgE levels were by far the strongest indicators associated with resolution. Similar to our study, a prospective study of 58 children in one center identified several factors associated with egg allergy resolution in multivariate analysis, including symptoms at the time of the reaction (strongest predictor), and eggspecific IgE. (13) Severity of initial reaction was also noted to relate to prognosis by Ford and Taylor. ${ }^{(14)}$ In a retrospective chart review analysis, Savage et al ${ }^{(11)}$ identified the highest recorded egg-specific IgE, presence of other atopic disease and other food allergies as predictive factors affecting resolution. These and other studies ${ }^{(32)}$ suggest, like ours, that elevated egg-specific IgE levels are strongly related to persistent egg allergy, a general concept which we also recently reported for milk allergy. ${ }^{(18)}$

Several additional factors that we identified as related to the natural course of egg allergy have been noted to be related to current/persistent egg allergy in a number of studies, including egg specific IgE/IgG4 ratios $^{(33)}$, and tolerance of egg in baked goods. ${ }^{(26 ; 34)}$ Regarding egg-specific IgG4, we might have expected higher IgG4 levels to predict resolution, as observed in immunotherapy treatment trials, ${ }^{(15)}$ but we saw the opposite, which may indicate a difference in mechanisms between natural tolerance and desensitization induced by immunotherapy. Egg specific IgG4 increases with exposure and the lower values in those who later developed tolerance could simply reflect earlier careful avoidance of larger exposures. Nonetheless, the relationship of IgG4 to outcomes was weak and not significant in multivariate analysis. Regarding ingestion of baked egg, we did not mandate exposure in this observational study, and did not begin to monitor baked egg ingestion until after baseline visits. While eventual ingestion of baked egg was associated with resolution, this outcome could reflect a milder phenotype prone to resolution, an immunotherapeutic benefit or a phenomenon related to accelerated testing with whole egg exposure after successful ingestion of baked egg. The distinctions are not evaluable in the present study design. Interestingly, among those with persistent allergy, exposure to baked egg products was not predicted by baseline characteristics.

T cell stimulation studies identified several markers associated with the natural course of egg allergy in our cohort. We found reduced expression of egg-specific IL-4 mRNA to be associated with resolution, consistent with one prior report. ${ }^{(35)}$ The subgroup evaluated here (those with egg allergy evaluated prospectively for resolution) are distinct from the entire cohort where we reported no IL-4 signal distinguishing those with or without baseline egg allergy/sensitization,(16) which may account for the different results. Nonetheless, the IL-4 
signal p-value was marginal and not a strong contributor to the predictive models. However, a number of IL4 responses to other stimulants were also associated with egg allergy prognosis, possibly reflecting a more generalized immune reactivity as a marker of resolution. Ultimately, these cellular studies did not contribute substantially to predicting outcomes.

Overall, our study has identified a number of factors that predict egg allergy outcomes, confirming a number of factors identified previously, and uniquely providing the opportunity to use our large, multicenter cohort to evaluate the most impactful baseline factors. The substantial predictive capacity of egg-specific $\operatorname{IgE}$ and clinical presentation allowed for the development of a novel algorithm to estimate the natural course of egg allergy. This composite index has been developed into an equation that can be applied to young $(<15$ months) patients presenting to the clinic and has been provided as a web-based calculator as well as a computer application (www.cofargroup.org). This unique tool may benefit healthcare providers and patients in providing early guidance as to the likelihood for disease resolution or persistence, but the utility of the calculator, although developed on a diverse clinical cohort, will need to be validated in other settings..

The strengths of this study include the sample size, the prospective design with re-evaluation at regular intervals, the inclusion of multiple research sites, and the exceptional follow-up rate. In addition, this study was the first to include detailed analysis of egg-specific IgG4 as well as T cell cytokine responses. Our results, similar to other natural history studies, ${ }^{(11-14)}$ are somewhat limited by the fact that OFCs were not performed at protocol-defined intervals in this observational study and therefore resolution rates are likely conservative estimates, and that many children were deemed egg tolerant based on unsupervised home introductions. Additionally, the study cohort was enrolled based on likely egg or milk allergy without known peanut allergy, which may distinguish this group from other clinical cohorts, although most infants prone to egg allergy would present to allergists with exposure to egg prior to having ingested peanut. ${ }^{(36)}$ In addition, the reliability of our algorithm may differ if different methods are used for IgE measurements, and, ultimately, will require validation in other settings.. An additional limitation was that we did not characterize bakedegg consumption in a rigorous manner, although approximately a third of those designated as egg allergic in our cohort reported consumption of products with baked-egg without a reaction at 6 years of age. It is important to recognize that our overall estimate of resolution does not include at least some children who might be fully tolerant of even whole forms of egg, or whether the introduction of baked-egg may have influenced the natural course of egg allergy in this cohort. Nonetheless, the study did not specifically encourage trialing or OFC to baked egg products, and so we believe the results reflect clinical practice.

In conclusion, we estimate from this well characterized cohort that approximately $50 \%$ of children with egg allergy will become egg tolerant by 6 years of age. Resolution is highly associated with lower egg-specific IgE levels, and the absence of systemic reactions beyond the skin on presentation. These highly predictive variables have been utilized to create a calculator to predict the natural history of egg allergy for individual patients, although additional studies to validate the model will be needed.

\section{Supplementary Material}

Refer to Web version on PubMed Central for supplementary material.

\section{Acknowledgments}

Sources of support: NIH-NIAID U19AI066738 and U01AI066560. The project was also supported by Grant Numbers UL1 TR-000154 (National Jewish), UL1 TR-000067 (Mount Sinai), UL1 TR-000039 (Arkansas), UL1 
TR-000083 (U North Carolina) and UL1 TR-000424 (Johns Hopkins) from the National Center for Research Resources (NCRR), a component of the National Institutes of Health (NIH). Its contents are solely the responsibility of the authors and do not necessarily represent the official view of NCRR or NIH.

Additional Site Investigators: FM Atkins, DYM Leung, TT Perry, AM Scurlock.

Coordinators and support: D Brown, L Talarico, S Noone, K Mudd, S Knorr, P Steele, J Kamilaris, S Carlisle, P Mayfield, M Mishoe, M. Beksinska, H Haczynska, J Grabowska, A Hiegel, June Straw, L Christie, M Groetch, J Ellingson, J Stone, S Leung, K Morgan, K Brown-Engelhardt, and S Cushing.

We thank Dr. Marshall Plaut, the medical officer, and J Poyser, for managing the project for CoFAR (NIAID) and Dr. R Lindblad from EMMES. We thank the families who kindly participated. We thank the staff of the clinical research units at each institution and the Statistical and Clinical Coordinating Center, without whose participation the study could not have been done. We thank Greer (Lenoir, NC) and Thermo Fisher Scientific (Waltham, MA) for supplying reagents. We would like to dedicate this work to our colleague and friend, Lloyd Mayer, MD.

\section{Abbreviations}

$\begin{array}{ll}\text { AD } & \text { atopic dermatitis } \\ \text { CoFAR } & \text { Consortium of Food Allergy Research } \\ \text { HR } & \text { Hazard ratio } \\ \text { OFC } & \text { oral food challenge } \\ \text { SPT } & \text { skin prick test }\end{array}$

\section{References}

1. Sicherer SH. Epidemiology of food allergy. J Allergy Clin Immunol. 2011; 127(3):594-602. [PubMed: 21236480]

2. Rona RJ, Keil T, Summers C, Gislason D, Zuidmeer L, Sodergren E, et al. The prevalence of food allergy: a meta-analysis. J Allergy Clin Immunol. 2007; 120(3):638-46. [PubMed: 17628647]

3. Gupta RS, Springston EE, Warrier MR, Smith B, Kumar R, Pongracic J, et al. The prevalence, severity, and distribution of childhood food allergy in the United States. Pediatrics. 2011; 128(1):e9-17. [PubMed: 21690110]

4. Eggesbo M, Botten G, Halvorsen R, Magnus P. The prevalence of allergy to egg: a populationbased study in young children. Allergy. 2001; 56(5):403-11. [PubMed: 11350303]

5. Osborne NJ, Koplin JJ, Martin PE, Gurrin LC, Lowe AJ, Matheson MC, et al. Prevalence of challenge-proven IgE-mediated food allergy using population-based sampling and predetermined challenge criteria in infants. J Allergy Clin Immunol. 2011; 127(3):668-76. [PubMed: 21377036]

6. Gaffin JM, Sheehan WJ, Morrill J, Cinar M, Borras CI, Sawicki GS, et al. Tree nut allergy, egg allergy, and asthma in children. Clin Pediatr (Phila). 2011; 50(2):133-9. [PubMed: 21098525]

7. Soderstrom L, Lilja G, Borres MP, Nilsson C. An explorative study of low levels of allergenspecific IgE and clinical allergy symptoms during early childhood. Allergy. 2011; 66(8):1058-64. [PubMed: 21392037]

8. Du Toit G, Roberts G, Sayre PH, Plaut M, Bahnson HT, Mitchell H, et al. Identifying infants at high risk of peanut allergy: the Learning Early About Peanut Allergy (LEAP) screening study. J Allergy Clin Immunol. 2013; 131(1):135-43. [PubMed: 23174658]

9. Liu AH, Jaramillo R, Sicherer SH, Wood RA, Bock SA, Burks AW, et al. National prevalence and risk factors for food allergy and relationship to asthma: results from the National Health and Nutrition Examination Survey 2005-2006. J Allergy Clin Immunol. 2010; 126(4):798-806. [PubMed: 20920770]

10. Fleischer DM, Perry TT, Atkins D, Wood RA, Burks AW, Jones SM, et al. Allergic reactions to foods in preschool-aged children in a prospective observational food allergy study. Pediatrics. 2012; 130(1):e25-e32. [PubMed: 22732173]

11. Savage JH, Matsui EC, Skripak JM, Wood RA. The natural history of egg allergy. J Allergy Clin Immunol. 2007; 120(6):1413-7. [PubMed: 18073126] 
12. Dannaeus A, Inganäs M. A follow-up study of children with food allergy. Clinical course in relation to serum IgE- and IgG -antibody level to milk, egg and fish. Clin Allergy. 1981; 11:533-9. [PubMed: 7332999]

13. Boyano-Martinez T, Garcia-Ara C, Diaz-Pena JM, Martin-Esteban M. Prediction of tolerance on the basis of quantification of egg white- specific IgE antibodies in children with egg allergy. J Allergy Clin Immunol. 2002; 110(2):304-9. [PubMed: 12170273]

14. Ford RPK, Taylor B. Natural history of egg hypersensitivity. Arch Dis Child. 1982; 57:649-52. [PubMed: 7125683]

15. Burks AW, Jones SM, Wood RA, Fleischer DM, Sicherer SH, Lindblad RW, et al. Oral immunotherapy for treatment of egg allergy in children. N Engl J Med. 2012; 367(3):233-43. [PubMed: 22808958]

16. Sicherer SH, Wood RA, Stablein D, Burks AW, Liu AH, Jones SM, et al. Immunologic features of infants with milk or egg allergy enrolled in an observational study (Consortium of Food Allergy Research) of food allergy. J Allergy Clin Immunol. 2010; 125(5):1077-83. [PubMed: 20451041]

17. Sicherer SH, Wood RA, Stablein D, Lindblad R, Burks AW, Liu AH, et al. Maternal consumption of peanut during pregnancy is associated with peanut sensitization in atopic infants. J Allergy Clin Immunol. 2010; 126(6):1191-7. [PubMed: 21035177]

18. Wood RA, Sicherer SH, Vickery BP, Jones SM, Liu AH, Fleischer DM, et al. The natural history of milk allergy in an observational cohort. J Allergy Clin Immunol. 2013; 131(3):805-12. [PubMed: 23273958]

19. Rajka G, Langeland T. Grading of the severity of atopic dermatitis. Acta Derm Venereol Suppl (Stockh). 1989; 144:13-4. [PubMed: 2800895]

20. Kull I, Wickman M, Lilja G, Nordvall SL, Pershagen G. Breast feeding and allergic diseases in infants-a prospective birth cohort study. Arch Dis Child. 2002; 87(6):478-81. [PubMed: 12456543]

21. Leung DY, Nicklas RA, Li JT, Bernstein IL, Blessing-Moore J, Boguniewicz M, et al. Disease management of atopic dermatitis: an updated practice parameter. Joint Task Force on Practice Parameters Ann Allergy Asthma Immunol. 2004; 93(3 Suppl 2):S1-21.

22. Food allergy: a practice parameter. Ann Allergy Asthma Immunol. 2006; 96(3 Suppl 2):S1-68. [PubMed: 16597066]

23. American Academy of Pediatrics. Committee on Nutrition. Hypoallergenic infant formulas. Pediatrics. 2000; 106(2 Pt 1):346-9. [PubMed: 10920165]

24. Sampson HA, Gerth van Wijk R, Bindslev-Jensen C, Sicherer S, Teuber SS, Burks AW, et al. Standardizing double-blind, placebo-controlled oral food challenges: American Academy of Allergy, Asthma \& Immunology-European Academy of Allergy and Clinical Immunology PRACTALL consensus report. J Allergy Clin Immunol. 2012; 130(6):1260-74. [PubMed: 23195525]

25. Clark TG, Bradburn MJ, Love SB, Altman DG. Survival analysis part I: basic concepts and first analyses. Br J Cancer. 2003; 89(2):232-8. [PubMed: 12865907]

26. Leonard SA, Sampson HA, Sicherer SH, Noone S, Moshier EL, Godbold J, et al. Dietary baked egg accelerates resolution of egg allergy in children. J Allergy Clin Immunol. 2012; 130(2):47380. [PubMed: 22846751]

27. Sampson HA, Scanlon SM. Natural history of food hypersensitivity in children with atopic dermatitis. J Pediatr. 1989; 115:23-7. [PubMed: 2738792]

28. Bock SA. The natural history of food sensitivity. J Allergy Clin Immunol. 1982; 69:173-7. [PubMed: 6895758]

29. Hill DJ, Firer MA, Ball G, Hosking CS. Natural history of cows' milk allergy in children: immunological outcome over 2 years [see comments]. Clin Exp Allergy. 1993; 23(2):124-31. [PubMed: 8448679]

30. Kulig M, Bergmann R, Niggemann B, Burow G, Wahn U. Prediction of sensitization to inhalant allergens in childhood: evaluating family history, atopic dermatitis and sensitization to food allergens. The MAS Study Group. Multicentre Allergy Study. Clin Exp Allergy. 1998; 28(11): 1397-403. [PubMed: 9824413] 
31. Lemon-Mule H, Sampson HA, Sicherer SH, Shreffler WG, Noone S, Nowak-Wegrzyn A. Immunologic changes in children with egg allergy ingesting extensively heated egg. J Allergy Clin Immunol. 2008; 122(5):977-83. [PubMed: 18851876]

32. Shek LP, Soderstrom L, Ahlstedt S, Beyer K, Sampson HA. Determination of food specific IgE levels over time can predict the development of tolerance in cow's milk and hen's egg allergy. J Allergy Clin Immunol. 2004; 114(2):387-91. [PubMed: 15316521]

33. Okamoto S, Taniuchi S, Sudo K, Hatano Y, Nakano K, Shimo T, et al. Predictive value of IgE/ IgG4 antibody ratio in children with egg allergy. Allergy Asthma Clin Immunol. 2012; 8(1):9. [PubMed: 22676477]

34. Clark A, Islam S, King Y, Deighton J, Szun S, Anagnostou K, et al. A longitudinal study of resolution of allergy to well-cooked and uncooked egg. Clin Exp Allergy. 2011; 41(5):706-12. [PubMed: 21488997]

35. Noma T, Yoshizawa I, Aoki K, Yamaguchi K, Baba M. Cytokine production in children outgrowing hen egg allergy. Clin Exp Allergy. 1996; 26(11):1298-307. [PubMed: 8955579]

36. Green TD, LaBelle VS, Steele PH, Kim EH, Lee LA, Mankad VS, et al. Clinical characteristics of peanut-allergic children: recent changes. Pediatrics. 2007; 120(6):1304-10. [PubMed: 18055680] 


\section{Clinical implications}

Egg allergy resolution by school age may be predicted by early clinical manifestations and egg-specific IgE levels. 


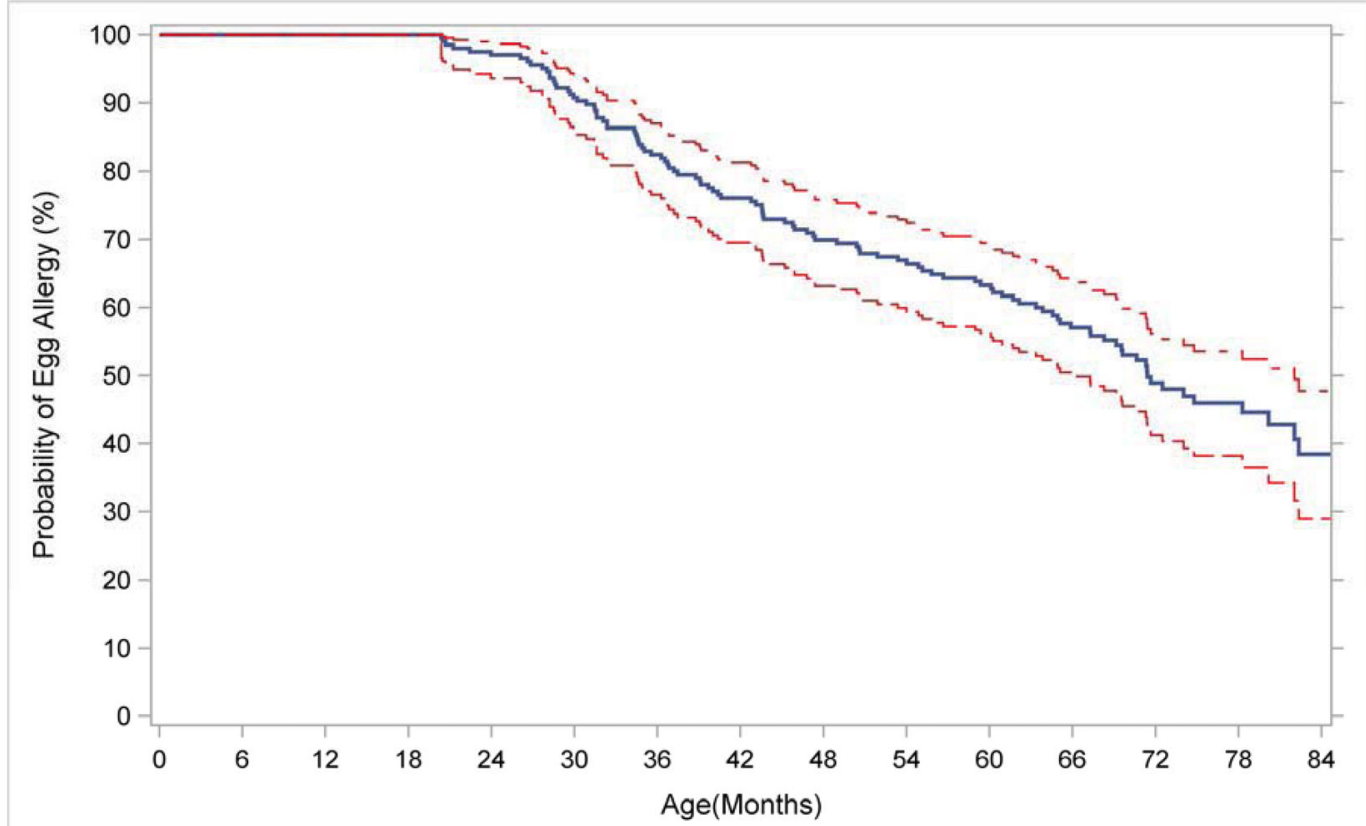

Figure 1.

Kaplan-Meier analysis of egg allergy resolution over time with point-wise $95 \%$ confidence intervals 


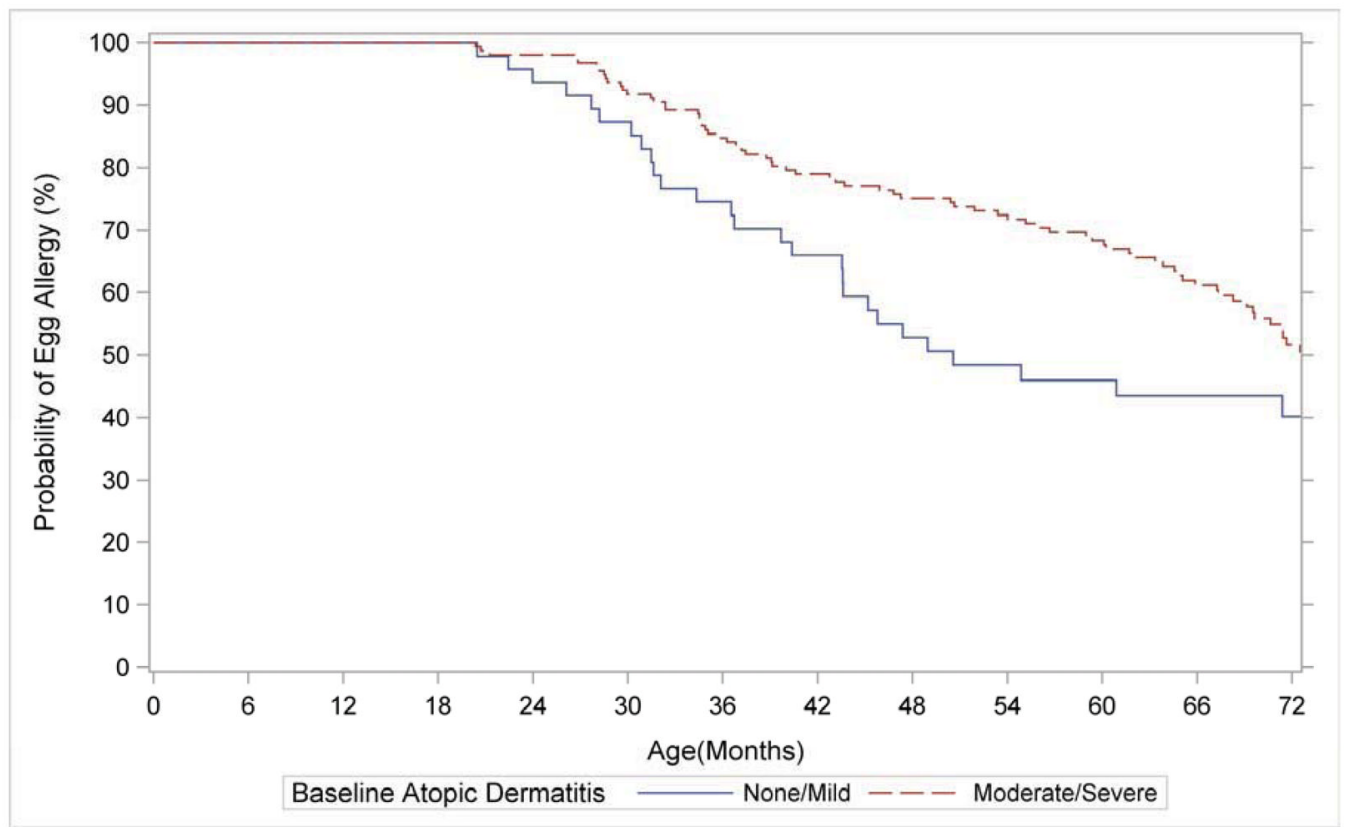

Figure 2.

Kaplan-Meier analysis representing the relationship of egg allergy resolution to baseline egg-specific IgE levels. Individual curves represent $\mathrm{IgE}$ levels of $<2 \mathrm{kU}_{\mathrm{A}} / \mathrm{L}$ (blue), $2-10$ $\mathrm{kU}_{\mathrm{A}} / \mathrm{L}$ (red), and $\geq 10 \mathrm{kU}_{\mathrm{A}} / \mathrm{L}$ (green). 


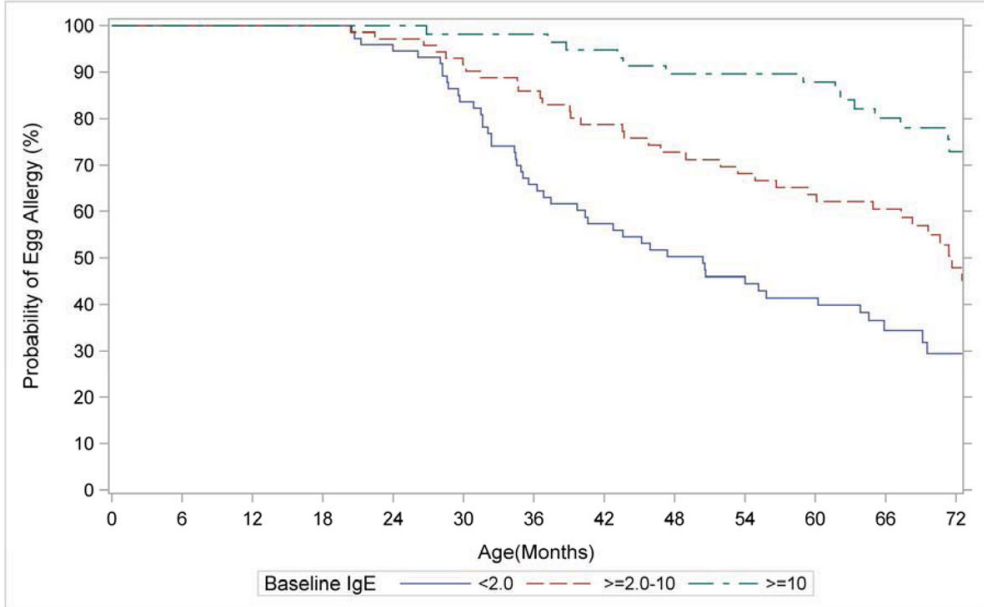

Figure 3.

Kaplan-Meier Analysis representing the relationship of egg allergy resolution to clinical presentation of initial reactions to egg. The mutually exclusive clinical presentation of the initial reaction to egg ingestion was categorized as the following: AD diagnosis (flare of $\mathrm{AD}$; this category included egg-IgE $>2 \mathrm{kU}_{\mathrm{A}} / \mathrm{L}$ ), skin only (acute hives and/or angioedema) or systemic (e.g., more than isolated skin, including respiratory and gastrointestinal reactions). 


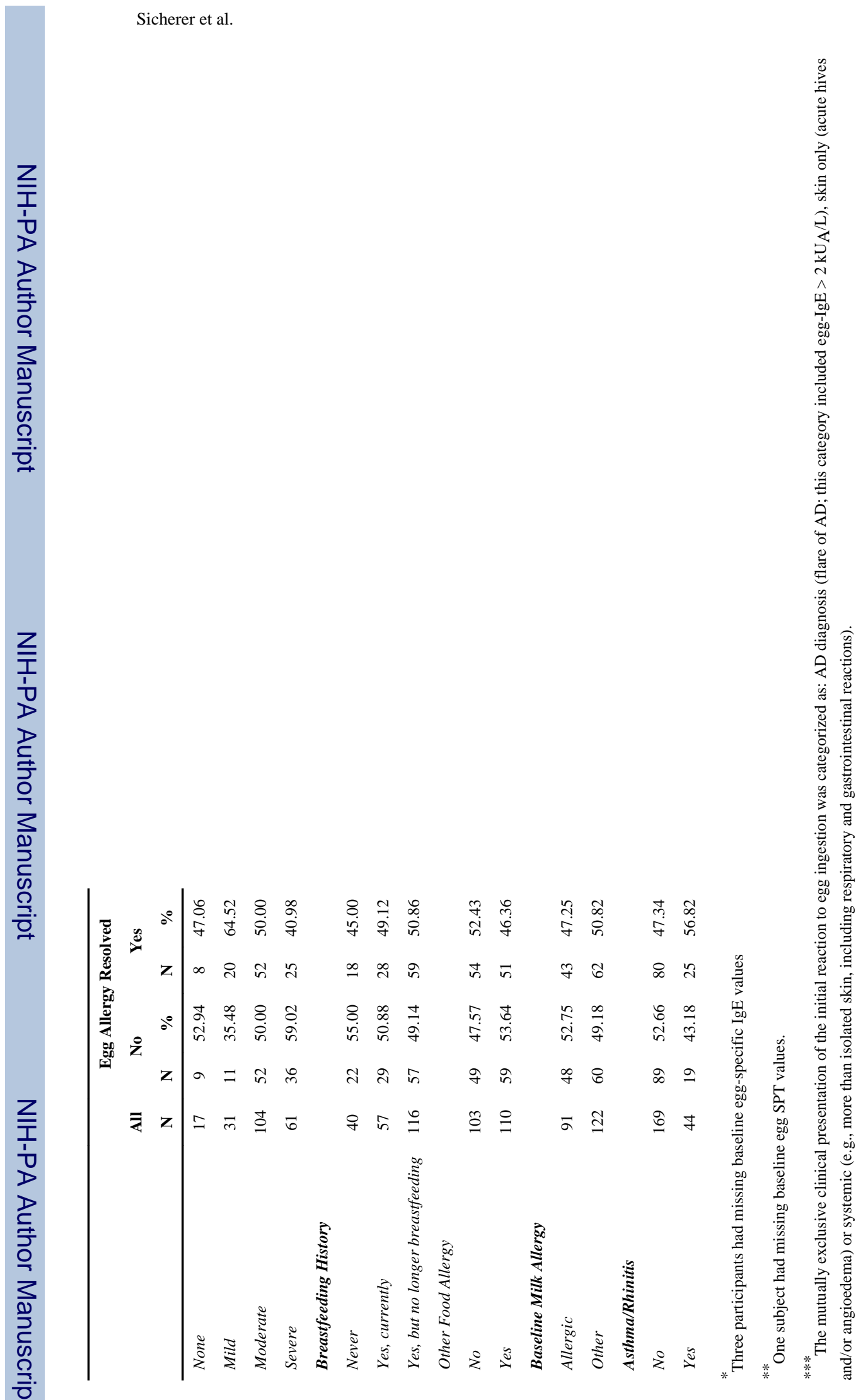

J Allergy Clin Immunol. Author manuscript; available in PMC 2015 February 01. 
Table 2

Resolution of egg allergy (Cox regression analysis with one variable in the model at a time)

\begin{tabular}{|c|c|c|c|}
\hline Risk Factor for Resolution of Egg Allergy & Hazard Ratio (HR) ${ }^{*}$ & 95\% HR Confidence Limits & P-value ${ }^{* *}$ \\
\hline \multicolumn{4}{|l|}{ Baseline Egg $\operatorname{IgE}\left(\mathbf{k U}_{\mathrm{A}} / \mathrm{L}\right)$ : } \\
\hline$\langle 2.0$ vs. $\rangle=10$ & 3.874 & $2.25,6.66$ & $<0.001$ \\
\hline $2-10$ vs. $>=10$ & 2.064 & $1.19,3.59$ & \\
\hline \multicolumn{4}{|l|}{ Baseline Egg SPT (wheal, mm): } \\
\hline$<5$ vs. $>=10$ & 1.995 & $1.23,3.24$ & 0.002 \\
\hline $5-<10$ vs. $>=10$ & 0.860 & $0.55,1.35$ & . \\
\hline \multicolumn{4}{|l|}{ Baseline Egg IgG4 (mgA/L): } \\
\hline$<0.10$ vs. $>0.4$ & 1.991 & $1.19,3.32$ & 0.022 \\
\hline $0.10-0.40$ vs. $>0.4$ & 1.346 & $0.78,2.33$ & . \\
\hline \multicolumn{4}{|l|}{ Baseline Age (months): } \\
\hline $3-5$ vs. $13-15$ & 0.821 & $0.32,2.08$ & 0.782 \\
\hline $6-8$ vs. $13-15$ & 1.189 & $0.68,2.07$ & . \\
\hline $9-12$ vs. $13-15$ & 0.907 & $0.59,1.40$ & . \\
\hline \multicolumn{4}{|l|}{ Sex: } \\
\hline Female vs. Male & 1.603 & $1.07,2.40$ & 0.022 \\
\hline \multicolumn{4}{|l|}{ Race: } \\
\hline White vs. Non-White & 0.941 & $0.60,1.49$ & 0.795 \\
\hline \multicolumn{4}{|l|}{ Baseline AD: } \\
\hline None/Mild vs. Moderate/Severe & 1.595 & $1.03,2.46$ & 0.036 \\
\hline \multicolumn{4}{|l|}{ Breastfeeding: } \\
\hline Yes, but no longer breastfeeding vs. Never & 0.811 & $0.48,1.38$ & 0.742 \\
\hline Yes, currently vs. Never & 0.846 & $0.47,1.53$ & . \\
\hline \multicolumn{4}{|l|}{ Other Food Allergy: } \\
\hline Yes vs. No & 0.737 & $0.50,1.08$ & 0.119 \\
\hline \multicolumn{4}{|l|}{ Asthma or Rhinitis: } \\
\hline Yes vs. No & 1.232 & $0.79,1.93$ & 0.364 \\
\hline \multicolumn{4}{|l|}{ Reaction Class: } \\
\hline Skin Only vs. Systemic Rx & 1.862 & $1.23,2.82$ & 0.007 \\
\hline AD Diagnosis vs. Systemic Rx & 0.961 & $0.48,1.93$ & . \\
\hline
\end{tabular}

* A Hazard Ratio > 1 indicates a proportional increase in chance of resolution of egg allergy. $* *$

p-value represents comparison of all variables in that category. 